\title{
DER BEGINN DER INSTITUTIONALISIERTEN LITERATUR IN UNGARN: \\ DIE GESELLSCHAFTEN DER INTELLEKTUELLEN IM MITTELALTER UND IN DER FRÜHEN NEUZEIT \\ Ein Forschungsbericht.
}

\author{
ANITA MARKÓ \\ Universität Wien und Eötvös Loránd Universität \\ anitamarko89@gmail.com
}

\begin{abstract}
Die ungarische Literaturgeschichte weist zahlreiche enge oder lose literarische Kreise und Schriftstellergruppen auf, die im 18. Jahrhundert den Beginn des modernen Literaturlebens kennzeichneten. Die bisherige Fachliteratur sagt, dass die ersten relevanten Zentren des ungarischen (und ungarisch geschriebenen) Literaturlebens erst in den schon hierarchisierten, akademischen und literarischen Kreisen und Salons im 18. Jahrhundert zu orten sind. Meine Forschungen führen mich hingegen zu der Erkenntnis, dass dieses Bedürfnis nach einem organisierten literarischen Leben schon im 15. Jahrhundert auftritt. Das Ziel meiner Arbeit ist es, den Beginn dieser frühen Gruppierungen, die sich von der der Aufklärungszeit natürlich unterschieden, zu erforschen. Die Forschung behandelt die Frage, welche Kontakte die Netzwerke des literarischen Lebens definierten und zuletzt was für ein Modell diese Kollektive gebildet haben. Mein Ausgangspunkt ist, diese frühen Gruppen als kulturelle Knotenpunkte zu sehen. Um den vorliegenden Quellenkorpus analysieren zu können, möchte ich die Methoden der Netzwerkforschung verwenden, und diese führt mich zu der Frage, wie wir diese Gesellschaften als ein Netzwerk, also in einem Graph visualisieren und analysieren können, um dadurch neue Informationen über die Wirkung und die Identität der Literaten zu erhalten. Mit diesem Forschungsbericht möchte ich die wichtigsten Überlegungen, den theoretischen und methodischen Rahmen des interdisziplinären Projekts darstellen.
\end{abstract}

Schlüsselwörter: Alte ungarische Literatur, Literarische Zusammenschlüssen, Netzwerk, Netzwerkanalyse, digital humanities

\section{Der Weg zu literarischen Zusammenschlüssen}

„Für jeden historisch denkenden Menschen bieten die verschiedenen Dichterbünde in der deutschen Literatur der letzten 500 Jahre ein höchst lehrreiches Anschauungsmaterial dafür, wie es selbst unter den gern als »Einzelgänger« apostrophierten Dichtern und Dichterinnen 
immer wieder zu Vereinigungen gekommen ist, die sich auf ihre Weise - nämlich durch das gedruckte Wort, die Theaterszene, die Rede oder den Anruf - in den Gang der politischen, sozialen oder kulturellen Entwicklung einzuschalten versuchen."1

Die Bedeutung der oben erwähnten Zusammenschlüsse von Poeten, Schriftstellern und anderen Intellektuellen bezieht sich nicht nur auf die deutschsprachige Literatur, die Erscheinung dürfte vielmehr ein allgemeines, grundlegendes Element dafür sein, dass wir die Wirkung des literarischen Lebens verstehen und analysieren können. Laut meiner These können diese Gruppierungen als Schlüsselformationen betrachtet werden, die zur institutionalisierten Literatur und zum Zustandekommen der literarischen Identität im Mittelalter und in der frühen Neuzeit geführt haben. Deshalb beabsichtige ich mit meiner Forschung die Tätigkeit der ungarischen Poeten- und Schriftstellergesellschaften im Mittelalter und im Zeitalter der Renaissance zu betrachten und beziehe mich unter anderem auf den Kreis um János Vitéz und István Radéczy. Ich möchte untersuchen, wie diese bekannten Schriftsteller und Poeten miteinander verbunden waren und in welcher Weise sie ihre Werke gegenseitig rezipierten.

Die ungarische Literaturgeschichte weist zahlreiche enge oder lose literarische Kreise und Schriftstellergruppen auf, die im 18. Jahrhundert den Beginn des modernen Literaturlebens kennzeichneten ${ }^{2}$. Die bisherige Fachliteratur sagt, dass die ersten relevanten Zentren des ungarischen (und ungarisch geschriebenen) Literaturlebens erst in den schon hierarchisierten, akademischen und literarischen Kreisen und Salons im 18. Jahrhundert zu orten sind ${ }^{3}$. Meine Forschungen führen mich hingegen zu der Erkenntnis, dass dieses Bedürfnis nach einem organisierten literarischen Leben schon im 15. Jahrhundert auftritt. Das Ziel meiner Dissertation ist es, den Beginn dieser frühen Gruppierungen, die sich von der Aufklärungszeit natürlich unterschieden, zu erforschen. Ich möchte analysieren, wie diese angenommenen literarischen Kreise in Ungarn funktionierten und stütze mich dabei auf ausländische Forschungsbeispiele. Da sich das Zeitalter, mit dem ich mich beschäftige, geopolitisch und kulturell auf weite Teile Europas erstreckt, gibt es auch viele Querverbindungen zwischen Wiener und ungarischen Denkern und Schriftstellern.

Die Forschungsfragen, die meiner Arbeit zugrunde liegen, sind die folgenden:

Von wann an finden sich die ersten Spuren solcher literarischen Zusammenschlüsse in Ungarn? Um dies beantworten zu können, muss ich als zweite Aufgabe feststellen, welche Kontakte die Netzwerke des literarischen Lebens definierten und zuletzt was für ein Modell diese Kollektive gebildet haben. Mein Ausgangspunkt ist, diese frühen Gruppen als kulturelle Knotenpunkte zu sehen. Um den vorliegenden Quellenkorpus analysieren zu können, möchte ich die Methoden der Netzwerkforschung verwenden, und diese führt mich zu der Frage, 
wie wir diese Gesellschaften als ein Netzwerk, also in einem Graph visualisieren und analysieren können, um dadurch neue Informationen über die Wirkung und die Identität der Literaten zu erhalten. Mit diesem Thema möchte ich an die derzeit laufenden Forschungen in Ungarn anknüpfen, die sich mit dem Beginn der ungarischen Literaturgeschichte beschäftigen. Ein weiterer Schwerpunkt meiner Arbeit ist es, den internationalen Kontext der Frage zu erforschen.

\section{Über den Korpus}

In meiner Forschung beabsichtige ich, mich gezielt mit der Epoche der ungarischen Literatur von ungefähr 1000 bis 1600 zu beschäftigen ${ }^{4}$. Denn um das Jahr 1000 definieren wir den Beginn einer kulturellen Formation, die wir schon als ungarisch oder hungarus bezeichnen können. Das von mir frei gewählte Enddatum erklärt sich wie folgt: Es spiegelt einerseits die Epochengliederung der ungarischen Quellenangaben wider, nach denen ich die Datensammlung ausführen möchte, andererseits steht es auch für eine natürliche Grenze der Machbarkeit. Denn ungefähr um 1600 begannen Druckereien in Ungarn zu wirken, somit würde der relevante Korpus in der Zeit danach immens anwachsen und man stünde Datenmengen gegenüber, die den Rahmen dieser Forschungsarbeit sprengen würden. Um 1600 starben zudem die bedeutendsten Kulturträger jener Zeit, Humanisten und Dichter wie Miklós Oláh (1568), István Radéczy (1586), Janos Zsámboky (1584), Bálint Balassi (1594).

Als ersten Schritt möchte ich einen konkreten und präzise umschriebenen Korpus mit Beispielen aus der frühen ungarischen und ausländischen Literatur erstellen, der das Gerüst meiner Dissertation bilden soll. Die primären Quellen werden anhand der ungarischen Quelleneditionen und Datenbanken Régi Magyar Költök Tára I-XII. (Sammlung der alten ungarischen Poeten, 16. Jahrhundert); Régi Magyar Könyvtár I-IV. (Alte Ungarische Bibliothek); Régi Magyarországi Nyomtatványok I. (Alte Ungarische Drucke); Magyar Müvelödéstörténeti Lexikon, Repertorium der alten ungarischen Gedichten (RPHA) festgelegt und beschrieben ${ }^{5}$.

Meine Forschung beschäftigt sich mit ungarischen literarischen Gruppierungen. In der so genannten alten ungarischen Literatur - wie auch für die meisten europäischen Kulturen zutreffend - bedeutet Literarität das Verfassen und Rezipieren von lateinischen Werken. Deshalb betrachte ich in der Arbeit Ungarisch nicht als sprachliche Grenze, sondern als einen verwaltungs- oder zugehörigkeitsmäßigen Rahmen: „Im Mittelalter können wir keinen subtilen Unterschied zwischen einem aus nationalem Gesichtspunkt betrachtet, ,ungarisch geboren' genannten Chronist, einem im Dienst von einem König aus dem Hause Árpád stehenden deutschen Hagiographen, einem kroatischen Humanisten von Hunyadis Hof und einem italienischen Geschichtsschreiber von König Matthias entdecken"6. 
Ich muss im Laufe der Bearbeitung des Themas auch Grenzen der geplanten Forschung definieren: Ich beschäftige mich mit schon publizierten Quellen, verarbeite Daten, die schon als Primär- oder Sekundärliteratur bekannt sind. Und ich muss darauf hinweisen, dass die Datenanalyse nie ein komplettes Bild ergeben kann, weil aus dieser Epoche nur wenige und oft zufällige Daten überliefert wurden. Mit diesem Problem kämpfen allerdings alle Forscher des Mittelalters: Wir müssen damit rechnen, dass neue Quellen und Daten, so sie entdeckt werden, Ergebnisse verändern können. Der Aufbau und die Verwendung einer Datenbank, die die Grundlage der Netzwerkanalyse bedeutet, bietet aber eine gute Möglichkeit dazu, immer neuere Informationen in die Forschungen einbringen zu können.

\section{Theoretische Begründung}

Was den Forschungsstand angeht, berücksichtige ich zwei wichtige Aspekte: Einerseits arbeite ich die Fachliteratur über die ungarischen literarischen Kreise durch, anderseits beschäftigte ich mich mit Analysen, die die Netzwerktheorie im Bereich der Literaturwissenschaft verwenden. Ich möchte zudem die Erörterung der Rezeption der internationalen und auch der ungarischen Fachliteratur fortsetzen. Der theoretische Hintergrund meiner Arbeit ist naturgemäß interdisziplinär: Neben philologischen Forschungsmethoden berücksichtige ich Zugänge der Soziologie, der Literatursoziologie und der Netzwerkforschung.

Die literatursoziologische und theoretische Forschung bestätigte, dass die Analyse der Netzwerkstruktur und der insgesamten Tätigkeit der mit obenstehenden Eigenschaften wirkenden literarisch-kulturellen Vereinigungen dazu beitragen könnte, besser zu verstehen, wie diese Schriftsteller und Poeten miteinander verbunden waren und ihre Werke gegenseitig rezipierten. Deshalb möchte ich literatursoziologische und netzwerktheoretische Thesen und Ansichtspunkte miteinander verknüpfen. Die wichtigsten Theoretiker für meine Arbeit sind Brian Stock, Pierre Bourdieu, Bruno Latour, Patrick Jagoda und Albert-László Barabási, Péter Csermely und Mark Buchanan? ${ }^{7}$ Wegen der Umfangsgrenze kann ich nur eine kurzgefasste Begründung dafür bringen, warum die Verknüpfung dieser Theorien in diesem Projekt anwendbar wäre. Zunächst halte ich den Begriff des literarischen Feldes von Bourdieu für einen geeigneten Ausgangspunkt in einer sich mit einem literatursoziologischen Thema beschäftigenden Forschung. Daneben ist auch das Werk von Howard S. Becker essentiell wichtig, der die Definition ,art world“ erstellt hat und unter diesem Begriff ,the network of people whose cooperative activity, organised via their joint knowledge of conventional means of doing things, produces the kind of art works that art world is noted for " versteht. Diese Theorien bieten eine Interpretation des Kunst-, beziehungsweise des literarischen Lebens, die sich durch Netzwerkanalyse belegen und begreifen 
lässt. Die Verwendungsformen der Netzwerktheorie in der Literaturwissenschaft hat Patrick Jagoda zusammengefasst. Er differenziert vier Bereiche: Historische Forschungen, den Gesichtspunkt der digital humanities („mapping“ und „modelling" der in und außerhalb der Texte wirkenden Strukturen), literatursoziologische Forschungen (Lesegesellschaften, Rezeptionsforschungen usw.), und formalistische Netzwerkanalyse des Textinhaltes. ${ }^{9}$ Mein Projekt sehe ich im dritten Bereich, der Literatursoziologie, verortet. Zusammen mit Jagoda (2014) gehe ich davon aus, dass ,With this method, networks serve not primariliy as tools, as they do in the digital humanities. Instead, they operate as social structures for imagining the precise circulation and reception of literary across communities, nations, and transnational contexts" $" 10$.

Für Kultur und Gesellschaft ziehe ich das Werk von Klaus P. Hansen ${ }^{11}$ heran. Er beweist, dass „Kultur... von Kollektiven getragen” wird. An die Stelle der soziologischen Begriffe Gruppe oder Gesellschaft setzt er den Begriff des Kollektivs: „Ein Kollektiv [...] liegt dann vor, wenn es mehr beinhaltet als die Summe seiner Mitglieder", und die Versatzstücke der individuellen Identität ,werden von Kollektiven angeboten, und die Individuen treten ihnen bei, wenn das Angebot ihren Neigungen entspricht." ${ }^{\prime 2}$ Latours Akteur-Netzwerk-Theorie bietet ebenfalls eine interessante Perspektive, weil er die humanen und nicht-humanen Akteure eines Netzwerkes nicht differenziert. So bekommt man ein hybrides Kollektiv, wo zum Beispiel nicht nur zwei Schriftsteller miteinander, sondern auch mit einem Buch oder einer Bibliothek in Verbindung gebracht werden können. Es ermöglicht eine interessante Interpretation des kulturellen Feldes von Bourdieu, und kann für die Epoche des Mittelalters - aus der wir wenige Autorennamen, aber mehrere Kodizes oder Wiegendrucke, also Titel kennen - geeignet sein. Die Forschung von Brian Stock sehe ich auch als wichtig, denn er erstellte die Definition der textual community, womit sich die Gesellschaft der Intellektuellen und das intellektuelle Feld im Mittelalter verknüpfen lassen.

Mit diesen Grundlagen der Literatursoziologie kann ich die Elemente der Netzwerktheorien von Barabási, Csermely und Buchanan (Kleine-Welt-Phänomen, Schwache Beziehungen, usw.) in dem Feld der mittelalterlichen litterae interpretieren.

\section{Überblick des Forschungsstandes}

Bereits eine meiner früheren Arbeiten handelte vom Zustandekommen und vom Wirkungsmodell solcher Gesellschaften. Ich habe die literarische Tätigkeit von János Vitéz bis zum Preßburger Kreis von Bischof Radéczy anhand der Quelleneditionen von Tibor Klaniczay und Ágnes Szalai Ritoókné analysiert ${ }^{13}$. Auf diese Arbeit sollen sich meine weiteren Forschungen stützen. Die zentrale Frage, 
mit der ich mich beschäftigt habe, war, ob die frühen literarischen Gruppen dem Inhalt des Begriffes „textual community“ gerecht werden ${ }^{14}$. Es geht dabei darum, wie sich diese Gemeinschaften selbst konstruieren hinsichtlich ihrer kollektiven und kulturellen Identität.

Die Zusammenschlüsse, mit denen ich mich unter Anwendung der erwähnten Fachliteratur beschäftigt habe, sind die oben erwähnten. Daneben kamen aber frühere Gesellschaften, wie die Literaten im Pannonhalma und um Bischof Mór (?1000-?1070) vor, oder andere Zusammenschlüsse, die die Wissenschaftler bis heute noch nicht als eine Gruppe oder Gesellschaft betrachten, die aber laut meiner Forschung auch durch Zusammenarbeit geprägt waren, wie zum Beispiel die Literaten um die Prediger Osvát Laskai (?1450-1511) und Pelbárt Temesvári (?1435-1504).

Die erste, von der Forschung als solche angesehene Gesellschaft ist die der Literaten und Humanisten um János Vitéz. Vitéz, Bischof von Nagyvárad (Großwardein), dann Erzbischof von Esztergom gilt als der erste Humanist in Ungarn. Fillippo Bounaccorsi nannte das Haus von Vitéz ,litterarum asylum”, und wir wissen, dass um 1440 in Buda, Várad oder Esztergom ein literarisches contubernium stattfand ${ }^{15}$, an dem vermutlich Gergely Szánoki, Philippo Podacataro, Pier Paulo Vergerio, Pál Ivanich und Giuliano Caeserini teilnahmen. Die Quellen können auch über fiktive Zusammenschlüsse berichten, denn Topoi dieser Art beweisen auch die Absicht des Zusammenschlusses, des so genannten literarischen Networking. 1467 fand in Esztergom ein weiteres symposion statt, dessen princeps, pater auch János Vitéz war. Unter den Teilnehmern finden wir Miklós Báthori, Janus Pannonius, Galeotto Marzio, Johannes Regiomontanus, Marcin Bylica, Giovanni Gatti und auch König Matthias Corvinus. Ugolino Verinos Epigramme und Conrad Keltis erwähnen auch eine Gesellschaft namens sodalis literraria, aktiv um 1470, mit den Teilnehmern (coetus) Miklós Báthori, Péter Garázda, János Váradi, Péter Váradi, Orbán Nagylucsei, Antonio Bonfini, Taddeo Ugoleto und Gulio Milio. Conrad Keltis gründete auch selbst eine derartige Gesellschaft: Seine Sodalitas Litteraria Danubiana kam 1479 zustande, Princeps des Budaer Zentrums könnte Janos Vitéz der Jüngere gewesen sein, und als die aktivsten Mitglieder können wir vier tschechische Humanisten benennen Augustinus Olomuciensis, Johannes Slechta, Georginus Neydecker und Julius Milius. Daneben waren noch einige ungarische Adelige unter den Mitgliedern: der Kanzler und Varader Bischof György Szathmári, der Veszprémer Bischof Gergely Frangepán, der Stuhlweißenburger Propst Zsigmond Thurzó, Fülöp Csulai Móré und Pál Várdai. Laut Fachliteratur kam es in den 1550-er Jahren in der Residenz des Pécser Bischofs György Szathmári György zu humanistischen Zusammenschlüssen, wie auch am Gyulafehérvárer Hof von Ferenc Várdai und am Hof von Erzbischof Miklós Oláh. 1580 bildete sich eine Gesellschaft von Studenten in Wittenberg namens Proles Palladis. Sie war durch einen Briefwechsel 
mit Iustus Lipsius verbunden, Mitglieder waren Mihály Forgách, Imre Forgách, Péter Révay és János Rimay. In der ungarischen Literaturgeschichtsschreibung gibt es eine jahrelange Debatte über einen vermeintlichen Dichterwettbewerb, dessen Spuren in einem Gedicht von Bálint Balassi lesbar sind. Der Poetenwettbewerb wird um 1588-89 datiert, die Akteure könnten vermutlich János Rimay, Ferenc Balassi, Jakab Dobó, Lőrincz Móricz, Mihály Kátay, György Enyedi und der von Balassi angestellte „Mátyás diák”, Pál Hunyadi Nagy oder Mátyás Nyéki Vörö ${ }^{16}$ gewesen sein. Obwohl es sich wahrscheinlich um einen fiktiven Anlass handelte, ist ein Zusammenschluss um Bálint Balassi leicht vorstellbar. Die Daten der geplanten Netzwerkanalyse werden vermutlich den Beweis für oder gegen die Existenz des Dichterwettbewerbs erbringen. Wir können noch kurz zwei Dichter erwähnen, die unter dem Einfluss von Janus Pannonius standen, nämlich Péter Garázda (1448-1507) und János Megyericsei (1470-1507), daneben die Kreise im Várad (1516-1522), deren Teilnehmer Sebestyén Magyi, László Debreceni, Fülöp Székesfehérvári (Philippus Alberegialus), Lőrinc Besztercei Ketschmer (Laurentius Bistricus), Bálint Hagymási (Valentinus Cybelius) waren. Auch die um Péter Károlyi wirkenden Kalvinisten und die Teilnehmer des Várader Religionsstreites (Mihály Varsányi, János Beregszászi, János Bonifác Debreceni, János Vetési, Péter Lörinc Beregszászi) könnten eine Form von Zusammenschluss gebildet haben. Der Kreis (1570-80) um Bischof István Radéczy kann bereits als Modell dienen: Die Mitglieder trafen einander regelmäßig im Garten oder in der Bibliothek von Radéczy. Sie schrieben Gedichte aneinander. Als Mitglieder der Gesellschaft, also als unanimi sodales - wie Radéczy sie nannte - galten der Geschichtsschreiber und Dichter Vicepalatin Miklós Istvánffy, der Humanist Elias Corvinus, der Preßlauer Arzt György Purkircher, der flandrische Philologe Nicasius Ellebodius ${ }^{17}$, der Humanist János Zsámboky und der Botaniker Carolus Clusius.

Laut meiner Hypothese beweisen die Topoi und Motive die Existenz solcher Gesellschaften, deren Mitglieder nicht nur innerhalb ihrer gesellschaftlichen Rollen wirkten, sondern sich daneben auch bewusst neue Identitäten aufbauten. Die Mitglieder erschienen nicht als geistliche (kirchliche) oder staatliche Positionsträger, sondern als sich mit Literarischem und Wissenschaftlichem beschäftigende Personen ${ }^{18}$.

Die beliebten Topoi und Motive dieser Gruppen waren die folgenden: Die wichtigste Eigenschaft war das Gruppenbewusstsein, die bewusste Repräsentation der literarischen Arbeit, die Beschäftigung mit litterae. Das Zusammengehörigkeitsgefühl war geprägt von „,nostra turba”, „,nostro censu”, „vatum caterva”. Manche Attribute sind quasi rituale Motive, die eine nahezu durchhierarchisierte Wirkung widerspiegeln: der feste Name (Sodalitas Litteraria Danubiana, proles Palladis), symbolische Dinge/Pflanzen (der Pokal von Olomuciensis, die Linde von Radéczy), die italienischen Tituli (princeps, pater, sodales). Dass die 
Gesellschaften als textual community handeln, ist auch zu betonen. Die Motive spiegeln ein Gesellschaftsmodell, in dem der Grund für den Zusammenschluss im gemeinsamen Wissen (par doctrina) und im gemeinsamen Handeln rund um den Text liegt.

Zur Erweiterung und Vertiefung der vorliegenden Forschungen wäre grundlegend der Begriff der „literarischen Gruppe“ für diese Epoche zu definieren. Zunächst arbeite ich an der Definition: Was bedeutet literarische Gruppe im Mittelalter und welches sind die Kontakte, die die vermeintlichen Netzwerke verbinden? Die Untersuchung der Tätigkeit der Poeten- und Schriftstellergesellschaften in der ungarischen Renaissance wirft eine wichtige Frage auf, die auch in der internationalen Fachliteratur noch nicht erschöpfend beantwortet wurde. Ein Schwerpunkt meiner Arbeit wäre demnach, auch den internationalen Kontext der Frage zu erforschen, und dazu benötige ich eine Definition der „literarischen Gruppen“, der auch auf frühe literarische Gesellschaften anwendbar ist. Bezüglich meiner Forschungsepoche gilt es festzuhalten, dass allein schon die üblichen Definitionen von Literatur und Gruppe (wie „Gesellschaft“, „Kreis“, usw.) nicht präzise und zutreffend sind.

\section{Literarische Gruppe? - Eine Attributenskala als Definition}

Einer der wichtigsten Teile der Forschung ist die Definitionsfindung. Um literarische Gruppen analysieren können, brauchen wir einen klaren und konsequenten Begriff von literarischen Zusammenschlüssen, den ich bisher weder in der ungarischen noch in der internationalen Fachliteratur gefunden habe. Deshalb sehe ich die Definitionsbildung als ein grundlegendes Element meiner Arbeit.

Die Bedeutung des Begriffs Literatur war im Mittelalter und auch später noch im Sprachgebrauch wesentlich breiter gefasst als in modernen Epochen, und der literarische Schaffensprozess war eine nach Regeln und Mustern erlernbare Kunst. In dieser Hinsicht sind sich die ungarische und internationale Fachliteratur einig ${ }^{19}$. Die bereits verarbeiteten Materialien zeigten aber, dass die Erfassung des Begriffs der Literarischen Gruppe noch viel komplizierter ist. Es gibt zwar Forschungen, die sich mit dem Thema beschäftigten ${ }^{20}$, verschiedene Zusammenschlüsse deutscher Autoren zu Dichterbünden oder andere Formen literarischer Gesellschaften wurden in zahlreichen detaillierten Einzelstudien analysiert, aber es gibt keine historisch umfassende Gesamtdarstellung ${ }^{21}$. Das Problem liegt darin, dass neuere Studien, die sich an eine Definition heranwagten, damit nur das moderne Literaturleben beschreiben, und die Studien, die über das Mittelalter oder die Renaissance schreiben, keinen klaren Begriff verwenden ${ }^{22}$.

Insgesamt zeigt sich, dass die frühen, literarisch aktiven Gesellschaften einen Begriff brauchen, der die Vielfalt der informellen Kommunikationsformen in 
Gruppen nicht einschränkt. Eine solche Definition müsste folgende differenzierende Gruppierungsgrade berücksichtigen: klare Leitziele, dichtungstheoretische Programme, ausgeprägter Sinn für Solidarität, innere Konsistenz, bestimmter Ort, zentrale Persönlichkeit, gemeinsame Anschauungen über Formen und Inhalte oder familiärer, freundschaftlicher oder auf Gläubigkeit beruhender Zusammenschluss. Eine meiner derzeitigen Aufgaben ist die Festlegung der Attribute, die der Definition zugrunde liegen können.

\section{Netzwerkanalyse in der Literaturwissenschaft: Methode der geplanten Forschung}

„,Networks influence practically every subfield of literary studies. “23

Als dritten großen Teil sehe ich die Beschreibung des Korpus und der Analysemethode, wobei hier die Methode der Netzwerkanalyse gemeint ist. Dazu werde ich ein Datenmodell und eine Datenbank erstellen, damit ich die Visualisierung und Analyse der zu berücksichtigenden Gesellschaften durchführen kann.

Mein Ziel ist es, eine umfassende Forschung über frühe Literaturkreise und Schriftstellergruppen durchzuführen. Als Grundlage für die Netzwerkanalyse plane ich die Erstellung einer Datenbank auf Basis der oben erwähnten Quelleneditionen. Die Datenbank sollte anhand der verschiedenen Kontakttypen entstehen, die den Begriff der Literatengesellschaften definieren. Die Zusammenführung der geordneten Datenmenge betrachte ich als das zweite angestrebte wichtige Ergebnis der Forschung, weil wir damit eine Datenbank schaffen können, die nicht nur die Kontakte der literarischen Gesellschaften aufzeigt, sondern auch andere Kreuzungspunkte und Zusammenschlüsse des kulturellen Feldes sichtbar macht. Zusammenhängende Daten lassen sich - einmal erfasst - mit der Methode der Netzwerkanalyse interpretieren ${ }^{24}$.

In den vorigen Jahrzehnten wurde Netzwerk zum Interpretationsrahmen und Beschreibungsmodell in zahlreichen wissenschaftlichen Disziplinen (Politikwissenschaft, Soziologie, Psychologie, Informatik oder Biologie). Im 21. Jahrhundert ist das Interesse für die Netzwerkforschung daher auch rasch gewachsen. Die Ursache war die Erkenntnis, dass die Struktur, die Herausbildung und die Entwicklung der in den komplexen Systemen wirkenden Netzwerke durch gemeinsame Grundgesetze geleitet werden ${ }^{25}$. Deshalb kann man alle diese Netzwerken mit interdisziplinären - graphtheoretisch-mathematischen, also empirischen und rechentechnischen Instrumenten erforschen, untersuchen.

Der Begriff des Netzwerkes wurde als Metapher oder Interpretationsmöglichkeit in der Literatur in den ersten Jahrzehnten des 21. Jahrhunderts verwendet. Dies hängt mit dem Erscheinen der neuen Medien zusammen. In allen Epochen 
der Literaturgeschichte wurden solche Netzwerke - Netz der Figuren in einem Text oder Netzwerke der Intertextualität vermerkt.

Die Verwendungsformen der Netzwerktheorie in der Literaturwissenschaft hat Patrick Jagoda ${ }^{26}$ zusammengefasst. Er unterscheidet vier Bereiche der netzwerkanalytischen Denkweise: die historische, digitale, soziologische und formalistische Methode. Die Bereiche sind schwer voneinander zu trennen, aber diese Richtungen bieten eine Interpretation des Kunst-, beziehungsweise des literarischen Lebens, die sich durch Netzwerkanalyse belegen und begreifen lässt.

Reale Netzwerke aus den verschiedensten Bereichen weisen häufig sehr ähnliche Eigenschaften und Strukturen auf. Ein Netzwerk besteht aus einer Menge von Elementen (Knoten), die mittels Verbindungen (Kanten) miteinander verbunden sind. Soziologisches Netzwerk bedeutet zwischenmenschliche Beziehungen, in denen Punkte Personen bezeichnen, Linien ihre gegenseitigen Beziehungen.

Die Netzwerkanalyse nutzt mehrere Verfahren, mit denen sich soziale Netzwerke analysieren und systematisch und quantifizierend beschreiben lassen. Somit können die Maßzahlen helfen, komplexe Netzwerke zu verstehen. Dadurch werden die Gradbestimmung, Zusammenhangskomponente, Kantendichte, Zentralität und Prestige, Hierarchisierung, Soziales Kapital, Weak Ties (schwache Beziehungen) und strukturelle Löcher, Cliquen und Cluster in dem Netzwerk des literarischen Lebens in der Epoche analysierbar.

Die moderne Fachliteratur beweist, dass die Netzwerkanalyse in der philologischen Forschung gute Ergebnisse erzielen kann. Die Studie von Alexandra Antal beispielsweise behandelt das Netzwerk einer Zeitungsredaktion (Bécsi Magyar Hírmondó) im 18./19. Jahrhundert; Anna Bátori beschäftigte sich mit dem durch Intertextualität und Zitieren entstehenden Netzwerk des Wissens in den Werken von Pál Wallaszky ${ }^{27}$. Netzwerke früherer literarischer Gesellschaften wurden noch nicht unter diesem Gesichtspunkt analysiert. Es gibt auch mehrere internationale Forschungsprojekte, die die Netzwerkanalyse und die Graphvisualisation benutzte, zum Beispiel das Six Degrees of Francis Bacon ${ }^{28}$ Projekt visualisierte das Ego-Netzwerk von Francis Bacon. Das Projekt der Stanford Universität, Circulation of Knowledge and Learned Practices in the 17th-century Dutch Republic $^{29}$ beschäftigt sich mit den Korrespondenzen der niederländischen Gelehrten im 17. Jahrhundert.

\section{Die Beziehungen zwischen den Verfassern der Einträge in den ungarischen Stammbüchern zwischen 1500-1700}

Ich möchte nun ein eigenes Beispiel der Visualisationsmöglichkeit ${ }^{30}$ kurz vorstellen: Die Beziehungen zwischen den Verfassern der Einträge in den ungarischen Stammbüchern zwischen 1500-1700. Hierbei habe ich eine bestimmte Beziehung 


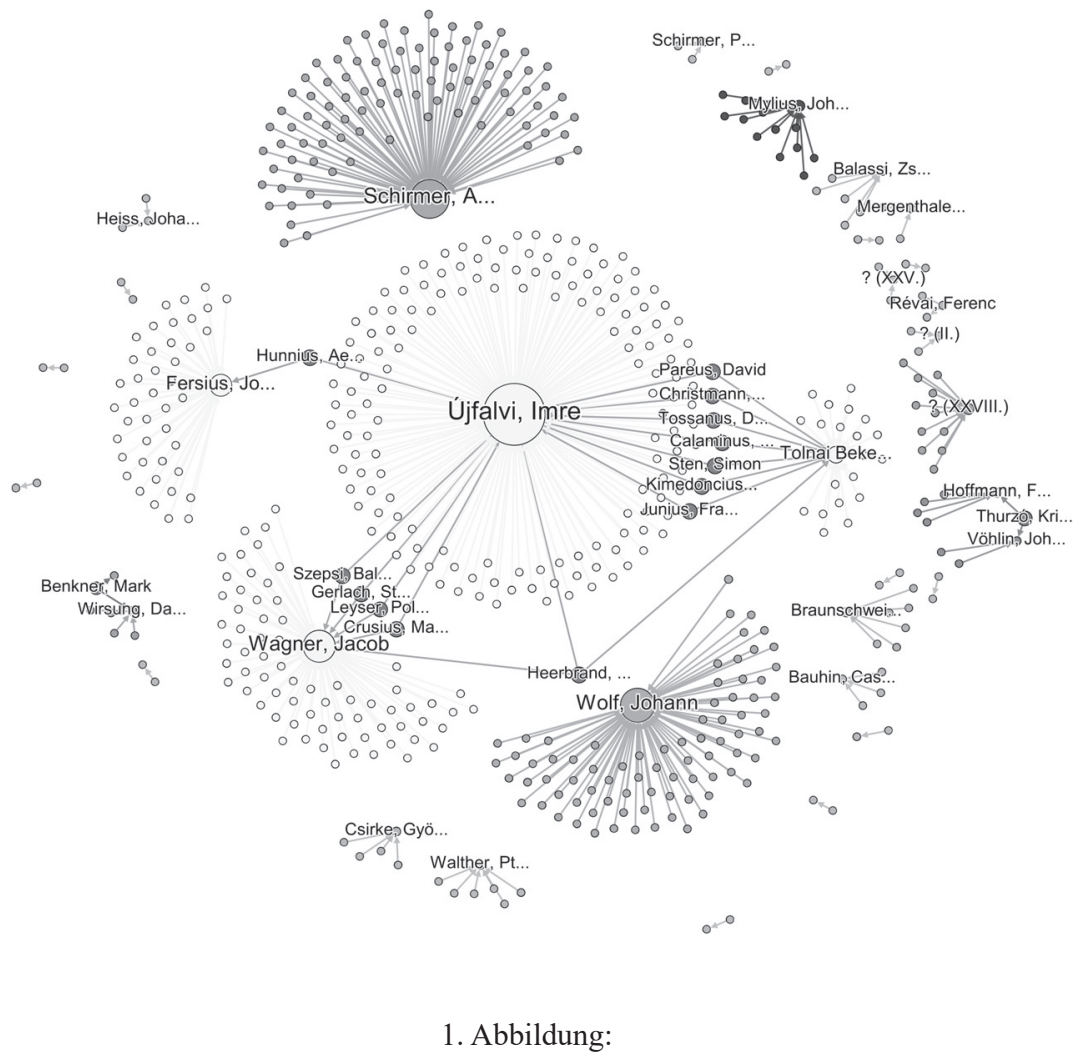

Die Beziehungen zwischen den Verfassern der Einträge in den ungarischen

Stammbüchern zwischen 1500-1600

in den Fokus gestellt, die Daten der ungarischen Stammbücher-Datenbank Inscriptiones Album Amicorum ${ }^{31}$ importiert und aufgearbeitet. Die Einträge der Stammbücher definieren die Kontakte des Netzes, für Knoten gelten die Verfasser und die Besitzer der Alben. Auf diese Weise ist ein Netzwerk definierbar, das aus 3682 Personen und 4080 Kontakten entsteht. Aus der Darstellung wird deutlich, dass die Mehrheit der Personen zu einer großen, dicht zusammengehörigen Komponente gehört. Wenn wir nun das erste Jahrhundert des Untersuchungszeitraums betrachten, ist das Wachstum des Netzwerkes deutlich sichtbar. Die meisten Komponenten, Teilnetzwerke wurden aus maximal 10 Personen aufgebaut, aber von den 70 Komponenten tauchte eine sogenannte ,giant component”, Riesenkomponente auf: Sie enthielt 3362 Personen, also mehr als 90\% der Teilnehmer. In der vorangehenden Epoche (1. Abbildung) können wir keine Clique oder Triade fin- 
den: Hier ist aber diese Zahl auf 132 gestiegen. (Die 3-Cliquen, Triaden bedeuten enge Freundschaftskreise, in denen drei Personen eine Beziehungen zueinander haben.) Dies bedeutet eine wichtige, qualitative Veränderung in der Struktur der Zusammenschlüsse. Wie man auf die 2. Abbildung sehen kann, lassen sich die Cliquen und die Vermittlerpersonen voneinander trennen. Die interessantesten Ergebnisse dieser Untersuchung sind, dass die wichtigsten Personen nicht die zentralen Albumbesitzer mit vielen Kontakten sind, sondern die topologisch in Vermittlerfunktion stehenden Figuren. Solche sind zum Beispiel die wittenbergischen Professoren, die die größeren Gruppierungen miteinander verknüpfen. Das Modell des Netzwerkes bietet eine gute Möglichkeit, die Inskriptionen der Alben neu kontextualisieren zu können.

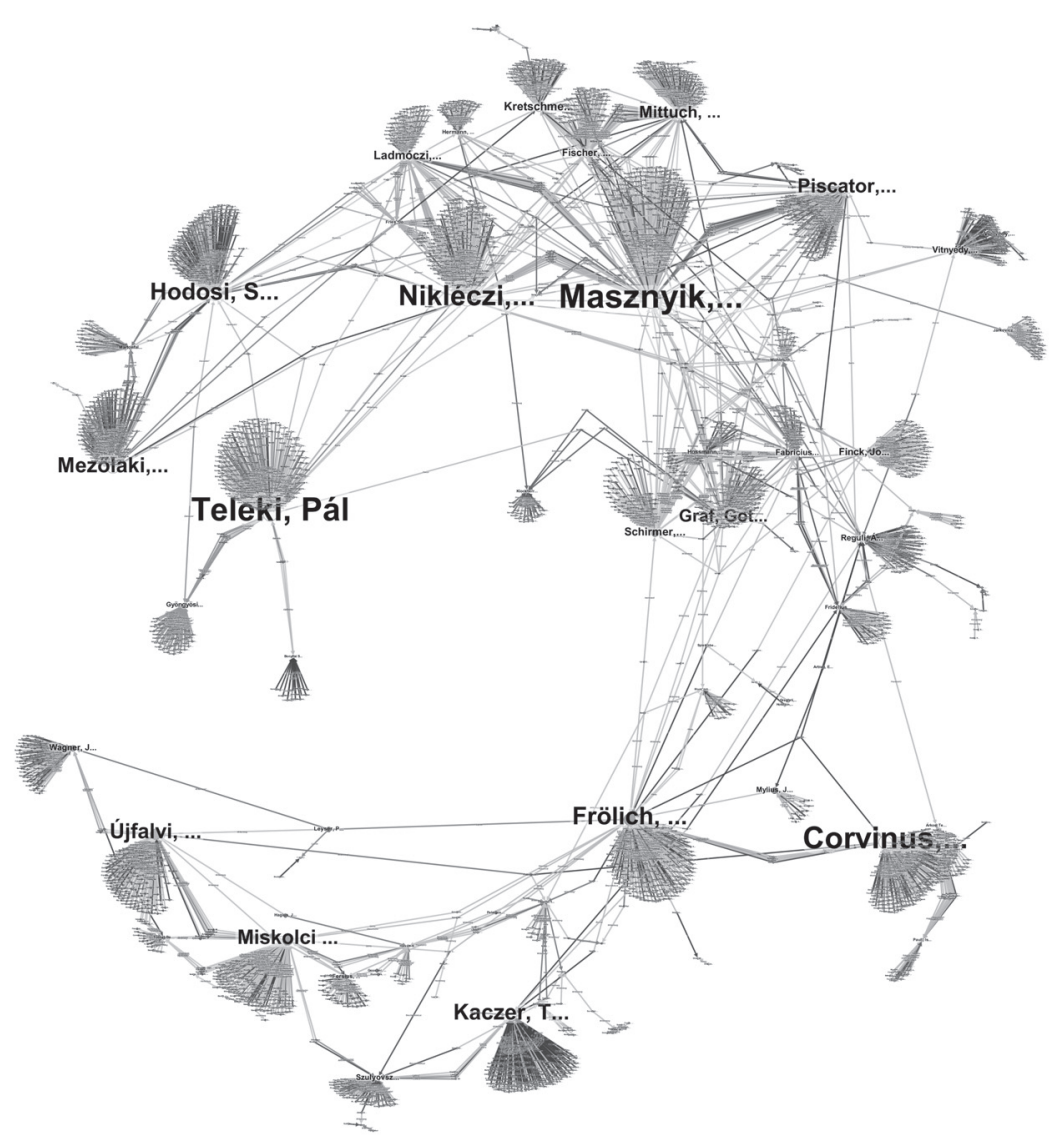

2. Abbildung:

Die Beziehungen zwischen den Verfassern der Einträge in den ungarischen Stammbüchern zwischen 1500-1700 (Riesenkomponente) 


\section{Weitere Schritte}

Die Beispiele zeigen, dass die Methode der Netzwerkanalyse interessante Ergebnisse erzielen kann: Es werden herausragende Personen und Teilnetzwerke, Zusammenschlüsse analysierbar, die sich in einer linearen Datenmenge verstecken können.

Die größte Aufgabe meines Projekts ist die Analyse der aus den Daten gewonnenen Knotenpunkte, also die Beschreibung der einzelnen ungarischen literarischen Gesellschaften, die im Zeitraum zwischen 1000 und 1600 belegbar auftraten und handelten.

Ich gehe davon aus, dass die hier beschriebene geplante Forschungsarbeit bedeutende Erkenntnisse in Bezug auf die alte ungarische Literatur und darüber hinaus auf ihre Auswirkungen auf die Literatur der späteren Epochen bringen kann. Denn die im Fokus stehenden und teils erst nachzuweisenden frühen Netzwerke haben zweifellos das weitere ungarische Literaturleben beeinflusst. Weitere, über dieses Projekt hinausgehende Forschungen können diese spezifischen Wirkungen der frühen literarischen Netzwerke analysieren, die sukzessive zum „modernen” literarischen Leben des 18. Jahrhunderts geführt haben.

\section{Anmerkungen}

1 Hermand, Jost: Die deutschen Dichterbünde: Von den Meistersingern bis zum PEN Club (Köln / Weimar / Wien: Böhlan Verlag, 1998), 5.

2 Klaniczay, Tibor (Hg.): A magyar irodalom története 1600-ig (Budapest: Akadémiai Kiadó 1964), 5.

3 Fábri, Anna: Az irodalom magánélete (Budapest: Magvető 1987), 7-9.

4 Über die umfassende Geschichte der Epoche: Engel, Pál: Szent István birodalma. A középkori Magyarország története (Budapest: História-MTA Történettudományi Intézete 2001), 343.; Szakály, Ferenc: Virágkor és hanyatlás 1440-1711 (Budapest: Háttér Lap- és Könyvkiadó 1990), 368.; Pálffy, Géza: A Magyar Királyság és a Habsburg Monarchia a 16. században (Budapest: História-MTA Történettudományi Intézete 2011), 564.; Mályusz, Elemér: Egyházi társadalom a középkori Magyarországon (Budapest: Akadémiai Kiadó 1971), 398.; Fügedi Erik: Uram, királyom... A XV. századi Magyarország hatalmasai (Budapest: Fekete Sas Kiadó 2004), 251.; Burckhardt, Jacob: A reneszánsz Itáliában (Budapest: Képzőmüvészeti Alap Kiadóvállalata 1978), 367.

5 Borsa, Gedeon u. a. (Hg.): Régi Magyarországi Nyomtatványok 1473-1600 (Budapest: Akadémiai Kiadó 1971); Horváth Iván u. a. (Hg.): Répertoire de la poésie hongroise ancienne I-II. (Paris: Le Nouvel Objet 1992). Digitale Ausgabe: Version 4.0, htttp://rpha.elte.hu/ 2017.05.01.; Régi magyar költők tára I-XII.; Köszeghy Péter (Hg.): Magyar müvelödéstörténeti lexikon VII. Középkor és kora újkor (Budapest: Balassi Kiadó 2007); Latzkovits, Miklós (Hg.): Inscriptiones Alborum Amicorum, 2003-2017, DOI: https://doi.org/ 10.14232/iaa.; Madas Edit (Hg.): „Látjátok feleim...” Magyar nyelvemlékek a kezdetektöl a 16. század elejéig. Az Országos Széchényi Könyvtár kiállitása (Budapest: OSZK 2009), 400.; Csapodi, Csaba u. a. (Hg.): Kódexek a középkori Magyarországon. Kiállitás: Az Országos Széchenyi Könyvtárban (Budapest: Interpress 1985), 295. 
6 Klaniczay, Tibor: Irodalom és nemzetiség. - In: Klaniczay, Tibor: Stílus, nemzet és civilizáció (Budapest: Balassi Kiadó 2001), 14. (Übersetzung von mir.)

7 Stock, Brian: The implication of Literacy. Written language and Models of Interpretation in the $11^{\text {th }}$ and $12^{\text {th }}$ centuries (New Yersey: Princeton University Press 1983), 604.; Csermely, Péter: A rejtett hálózatok ereje. Hogyan stabilizálják a világot a gyenge kapcsolatok? (Budapest: Vince Kiadó 2004), 376.; Buchanan, Mark: Nexus. Avagy kicsi a világ (Budapest: Typotech 2013), 239.; Barabási, Albert-László: Behálózva (Budapest: Magyar Könyvklub 2003), 320.; Barabási, Albert-László: A hálózatok tudománya (Budapest: Libri Kiadó 2016), 445.; Latour, Bruno: Reassembling the Social. An Introduction to Actor-Network-Theory (New York: Oxford University Press 2005), 301.; Bourdieu, Pierre: A müvészet szabályai. Az irodalmi mezó genezise és struktúrája (Budapest: Budapesti Kommunikációs és Üzleti Főiskola 2013), 368.; Jagoda, Patrick: Networks in Literature and Media. - In: Oxford Research Encyclopedia. Literature (USA: Oxford University Press 2017). DOI: 10.1093/acrefore/9780190201098.013.135, 05. 04. 2017.

8 Becker, Howard S.: Art world (Berkely/Los Angeles/London: University of California Press 1982)

9 Jagoda 2017, 7.

10 Jagoda 2017, 7-8.

11 Hansen, Klaus P.: Kultur und Kulturwissenschaft. Eine Einführung. 2. Auflage (Tübingen / Basel: A. Francke Verlag 2000), 157, 194, 195.

12 Hansen 2000, 195.

13 Ritoókné Szalay, Ágnes: Hortus Musarum. Egy irodalmi társaság emlékei. - In: Ritoókné Szalay, Ágnes: Nympha super ripam Danubii. Tanulmányok a XV-XVI. századi magyarországi müvelödés köréből (Budapest: Balassi Kiadó 2002), 219-223.; Ritoókné Szalay, Ágnes, Versek a Radéczy körböl. - In: Ritoókné Szalay, Ágnes: Nympha super ripam Danubii. Tanulmányok a XV-XVI. századi magyarországi müvelödés köréböl (Budapest: Balassi Kiadó 2002), 223-234.; Klaniczay, Tibor: A magyarországi akadémiai mozgalom előtörténete (Budapest: Balassi Kiadó 1993), 95.; Klaniczay, Tibor: Pallas magyar ivadékai (Budapest: Szépirodalmi Kiadó 1985), 353.; Pajorin, Klára: A humanista symposionrendezvények. - In: Karsay, Orsolya (Hg.): Uralkodók és corvinák. Az Országos Széchényi Könyvtár jubileumi kiállitása alapításának 200. évfordulójára (Budapest: OSZK 2002), 111-115.

14 Stock 1983, 604.

15 Klaniczay 1993, 30.

16 Vadai, István: "Balassi és Echo", Palimpszeszt, 10 (1998). Digitale Ausgabe: http://magyar-irodalom.elte.hu/palimpszeszt/10_szam/02.htm, 05.05.2018.

17 István Monok: „Ex libris Nicolai Bethlen et amicorum” Az ,et amicorum” bejegyzésröl és a közös könyvhasználatról. - In: Császtvay, Tünde / Nyerges, Judit (Hg.): Humanizmus és gratuláció. Szolgálatomat ajánlom a 60 éves Jankovics Józsefnek (Budapest: Balassi Kiadó 2009), 269.

18 Ich nutzte als die Grundlagen dieser Analyse das Werk von Gergely Fazakas über die Gesellschaftsbildung/auslegung: Fazakas, Gergely Tamás: Siralmas imádság és nemzeti önszemlélet. A lamentációs és penitenciás sírás a 17. század második felének magyar református imádságoskönyveiben (Debrecen: Debreceni Egyetemi Kiadó 2012), 444.

19 Ács, Pál: Reneszánsz irodalom. - In: Köszeghy Péter (Hg.): Magyar müvelödéstörténeti lexikon VII. Középkor és kora újkor (Budapest: Balassi Kiadó 2007), Digitale Ausgabe: MTA BTK ITI 2013-2016, http://mamul.btk.mta.hu/MAMUL_SZERK/mamul_view.php?editid1=5566, 01.05.2017.; Literatur. - In: Brockhaus Enzyklopädie Online. (München: Brockhaus NE GmbH; Gütersloh/München: F.A. Brockhaus, wissenmedia in der inmediaONE GmbH 2013-2015; Erscheinungsverlauf: 2013, März -). 


\section{DER BEGINN DER INSTITUTIONALISIERTEN LITERATUR IN UNGARN 333}

20 Einige der wichtigsten sind: Summers, Claude J./Pebworth, Ted-Lary (Hg.). Literary Circles and Cultural Communities in Renaissance England (Columbia: University of Missouri Press 2000), 243.; Campbell, Julie: Literary Circles and Gender in Early Modern Europe. A Cross-Cultural Approach (Aldershot: Ashgate Publishing Company 2006), 222.; Claessens, Dieter: Gruppe und Gruppenverbände. Systematische Einführung in die Folgen von Vergesellschaftung (Hamburg: Verlag Dr. Kovač 1995), 112.; Jost 1998, 383.; Kolk, Rainer: Literarische Gruppenbildung. Am Beispies de Georg-Kreises 1890-1945 (Tübingen: Max Niemeyer Verlag 1998), 697.; Wülfing, Wulf/Bruns, Karin/Parr, Rolf: Handbuch literarisch-kultureller Vereine. Gruppen und Bünde, 1825-1933 (Stuttgart/Weimar: Verlag J. B. Metzler 1998), 597.; Glowiński, Michaił: Literarische Gruppe und Poesiemodell. Das Beispiel der Gruppe Skamander. - In: Fieguth, Rolf (Hg.): Literarische Kommunikation (Kronberg/TS.: Scriptor Verlag 1975. S. 43-66.

21 Jost 1998, 1.

22 Siehe: Biró, Annamária/Boka, László (Hg.): Értelmiségi karriertörténetek, kapcsolathálók, írócsoportosulások (Nagyvárad/Budapest: Partium Kiadó/reciti 2014), 348.; Németh, Luca Anna: "Az irodalomi modernség egy szervezeti kísérlete: A Vörösmarty Akadémia története és szerepe a 20. század elejének irodalmi életében”, Filológia 2(2012), 48-79.; Kálmán C., György: "Dísz-funkciók: irodalmi társaságok a 20. század elején”, Partitúra 9(2014), 39-50.

23 Jagoda 2017, 1.

24 Im Zusammenhang mit den Operationen, dem Umgang mit quantitativen Daten und der Begriffen der oben erwähnten Maßnahmen siehe: Stegbauer, Christian, Häußling, Roger (Hg.): Handbuch Netzwerkforschung (Wiesbaden: Springer VS Verlag für Sozialwissenschaften 2010), 966.; Takács, Károly (Hg.): Társadalmi kapcsolathálózatok elemzése (Budapest: BCE Szociológiai és Társadalompolitikai Intézet 2011), 349. Digitale Ausgabe: https://www.tankonyvtar.hu/hu/tartalom/tamop425/0010_2A_08_Kapcsolathalo_elemzes_szerk_Takacs_Karoly 10010_2A_08_Kapcsolathalo_elemzes_szerk_Takacs_Karoly.pdf, 11.05.2018.

25 Barabási 2016, 42.

26 Jagoda 2017, 3.

27 Antal, Alexandra: “A bécsi Magyar Hírmondó (1789-1803) szerkesztői hálózata”, Irodalomtörténeti Közlemények, 118/1(2014), 99-117.; Bátori, Anna: “A tudás hálózatai. Wallaszky Pál historia litterariája és a 18. századi tudástranszfer”, Irodalomismeret, 3(2016), 35-63.

28 Warren, Christopher (Hg.), Six Degress of Francis Bacon (Carnegie Mellon UniversityGeorgetown University), http://www.sixdegreesoffrancisbacon.com, 05.05.2018.

29 Mijnhardt, Wijnand (Hg.), Circulation of Knowledge and Learned Practices in the 17th-century Dutch Republic, v. 1.0 (Haag), http://ckcc.huygens.knaw.nl/epistolarium, 05.05.2018.

30 Die Abbildungen und Netzwerkanalyse wurden mit der Software Microsoft Excel 2010 und Gephi (v. 0.9.2) gefertigt.

31 Latzkovits, Miklós (Hg.): Inscriptiones Alborum Amicorum (2003-2017), DOI: https://doi. org/10.14232/iaa, 05.05.2018. 